\title{
A Retrospective Study on "Effectiveness of Remote Patient Monitoring in Covid-19 patients"
}

\author{
Dr. Mallikharjuna Raj Kampally ${ }^{1}$, Dr. Mehdi Ali Mirza ${ }^{2}$, \\ Dr. Sony Agarwal ${ }^{3 *}$
}

${ }^{1}$ Research Associate-Clinical Affairs-Vigocare, Hyderabad, India

${ }^{2}$ ESIC Medical College and Hospital-Asst. Prof, Dept. of Pharmacology, Hyderabad-

India.

$3^{3 *}$ Lead-Medical Affairs-Vigocare, Hyderabad, India

Email:Mallikharjun.k@vigocare.com,Mehdialimirza@gmail.com,Sony.a@vigocare.com

\begin{abstract}
Corona virus disease19 has spread over the world, affecting millions of people. It has put enormous strain on the global healthcare system. Due to frequent mutations, the pandemic is spreading rapidly. The world requires a technology that will facilitate the effective diagnosis, treatment, and discharge of COVID19 patients. A model like remote patient monitoring [RPM] makes it easier to handle Covid 19 patients. RPM helps in remotely diagnosis, treatment, as well as allowing for prompt interventions. The RPM makes use of mobile technology and IoT platforms to take clinical interventions. In this study out of 151 covid19 positive subjects $91 \%$ of them were shifted to home monitoring within 5 days of MVM monitoring with few readmissions. The study investigated the effectiveness of RPM in the Indian healthcare system, as well as the performance and usability of the Vigocare mobile application by patients and doctors.
\end{abstract}

Keywords: Covid-19, Remote patient monitoring, management, mobile application

\section{Introduction}

The corona virus disease Covid 19 has become a global pandemic, and patient management has become a serious challenge [1][2]. With mutated variants, the covid 19 is becoming tougher by the day. High fever, shortness of breath, dry cough, and bodily pains are the most prevalent symptoms of the disease. The COVID19 virus infects the lungs, causing plaques to grow on the lungs and develops serious harm to the respiratory system. The infected patient's breathing becomes difficult [3]. Damaged lungs absorb oxygen inadequately, resulting in lower levels of dissolved oxygen in the blood [Spo2]. [4]. with new varieties such as Delta, Omicron, MU, and Omega, the COVID 19 virus has become more virulent. According to the WHO, Close-contact settings, especially where people conduct discussions very close together; confined and enclosed locations with poor ventilation are all important factors in the viruses transmission and rapid spread [5]. The 
new Omicron form could pose a greater threat to healthcare systems in the world's most populous countries, such as India. Low and middle-income nations have weak medical infrastructure, making it difficult to control or manage an outbreak of Covid 19 virus with variations like Omicron.

Healthcare's security and to manage the patients, physicians, nursing, diagnosis, and sanitization staff are essential. There is a demand for technology that can help to reduce of personal interaction with patients or one-on-one contact [6]. Remote patient monitoring is a technology that allows us to monitor a patient without having to touch them or have personal contact with them. Temperature, Spo2, heart rate, and respiration rate must all be monitored in order to examine and evaluate a patient's medical wellbeing [4]. A pulse oximeter, thermometer, and other devices are used to monitor the patient's vital signs. Dyspnea [55.4 percent], anxiety [55.4 percent], and inability to concentrate were the most commonly reported psychological problems through RPM.

According to a study from the United States. The most serious symptom was anxiety [9.8\%], followed by trouble concentrating [7]. [4.5 percent ]. According to another study, they were able to recruit 79.2 percent of patients in an interdisciplinary RPM programme for ambulatory remote patient monitoring programme for covid19 patients. The study's comments demonstrated a high proportion of patient satisfaction [8]. They created low and high intensity research models based on the severity of the symptoms for the study.

Similarly, the St Antonius Hospital in the Netherlands developed an RPM programme that used at-home oxygen saturation, temperature, and symptom tracking to track patients with severe COVID-19 after they were discharged from the hospital, including those who were getting oxygen therapy at home. The average period of hospitalizations was reduced by $5.0 \pm 3.8$ days per patient, and 97 percent of patients said the programme was easy to use [1]. Patients with COVID-19 were given a pulse oximeter after discharge, and one nurse was assigned to monitor 50 patients, according to the RPM programme at New YorkPresbyterian Brooklyn Methodist Hospital. In ten post-program questionnaires, patients expressed great levels of satisfaction, with over 91 percent saying they would suggest the programme to others[2]. A research on Secure Health Monitoring Communication Systems Based on IoT and Cloud Computing for Medical Emergency Applications was demonstrated high accuracy and reliability of the proposed system.[13]

Another study using the Mayo Clinic mobile app found that combining telehealth with remote patient monitoring and a mobile app offered better results. Especially for high-risk patients, with very few readmissions to hospital monitoring[8]. Remote monitoring via a smartphone application, in combination with a 24/7 live central monitoring platform, symptom tracing via tele conversation, and weekly doctor appointments, may yield positive and satisfactory results [9]. In nations with inadequate medical infrastructure, such as India, there is a need to apply and research the efficiency of RPM technology. In this regard, the present study aims to evaluate the efficiency of RPM using Vigo MVM solution. 


\section{Materials and Methods}

In nations with underdeveloped medical infrastructure, such as India, it is critical to deploy and research the effectiveness of RPM technology. Vigo Care has created a one-of-a-kind software-as-a-service platform that can link a pulse oximeter with a US-FDA-approved biosensor. Temperature, heart rate, and respiratory rate are all monitored via the FDAapproved vigo MVM Biosensor solution patch. The Spo2 Patch measures the Pulse Rate and Oxygen Saturation Levels.

\subsection{Pulse oximeter:}

A pulse oximeter is a device that measures blood oxygen levels in the form of saturation levels, which is an important vital sign for determining the patient's early warning score. The study was conducted using a clinically validated pulse oximeter.

\subsection{Vigo MVM Solution:}

Vigo MVM is an FDA-approved patch containing a biosensor and a unique patch ID that measures skin temperature, heart rate, and respiratory rates.

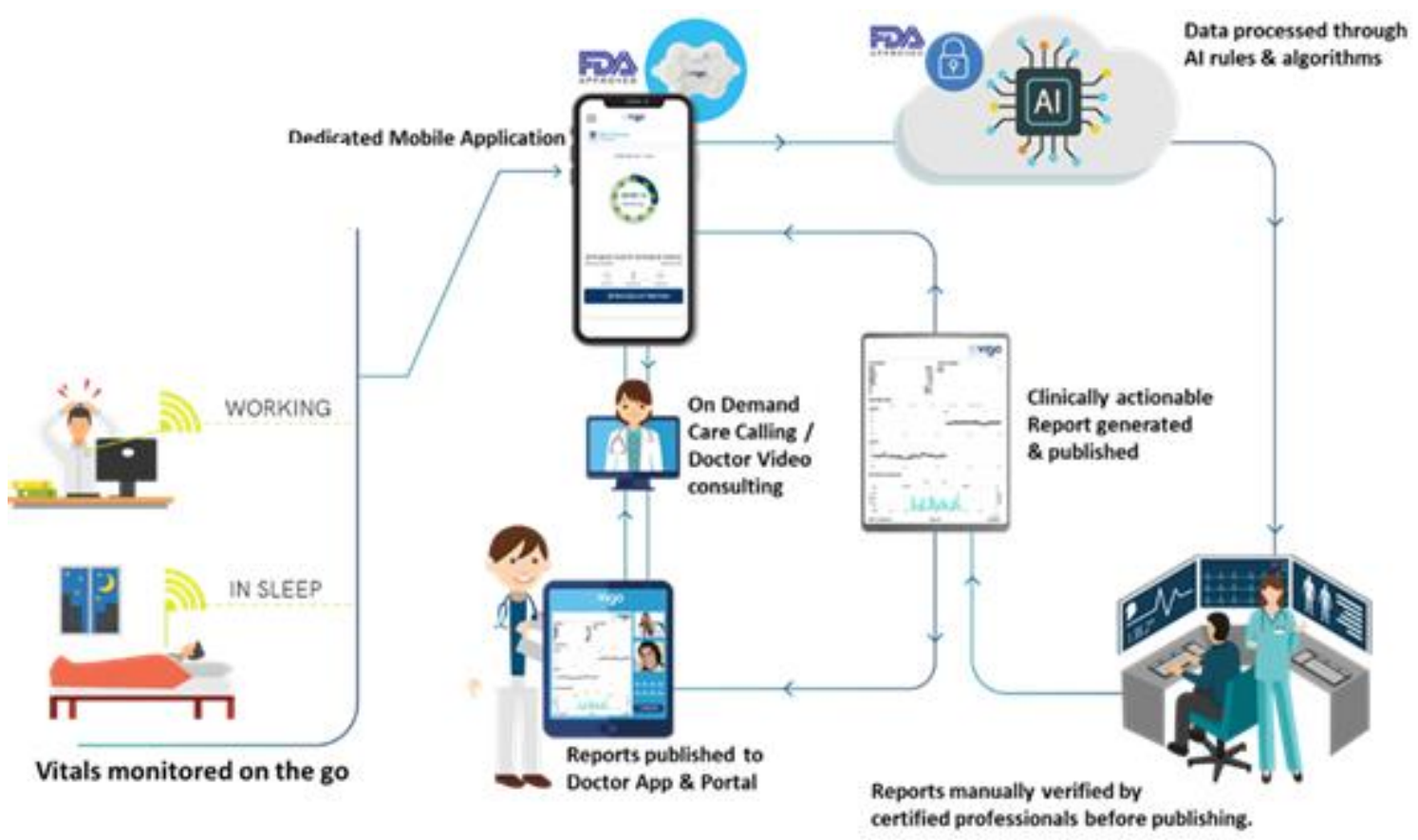

Fig1. Vigo Multi-Vital Monitoring [MVM] solution work-flow

2.3 Vigo care mobile application: a Vigo health care ltd-developed android mobile application that plays a critical role in remote patient monitoring.

\subsection{Onboarding and Operation}

Patient onboarding is a basic process with numerous steps in which the patient must download and connect the app to their mobile device. Through a hotspot, the mobile 
application links to a pulse oximeter and an FDA-approved Vigo solution patch. The Vigocare mobile application, which is based on Android, is accessible in the Google Play Store and must be downloaded and installed on a mobile device. Following installation, the patient must follow the on-screen instructions to link the vigo MVM solution patch to the mobile application. Every MVM patch has a four-digit code that is unique. The MVM patch was attached after inputting a 4-digit number in the mobile application. The software continuously captures vital information and uploads it to a cloud.

The vitals patterns were analysed by the Vigo engine, which automatically marked the trends with different colour codes based on the readings and severity.

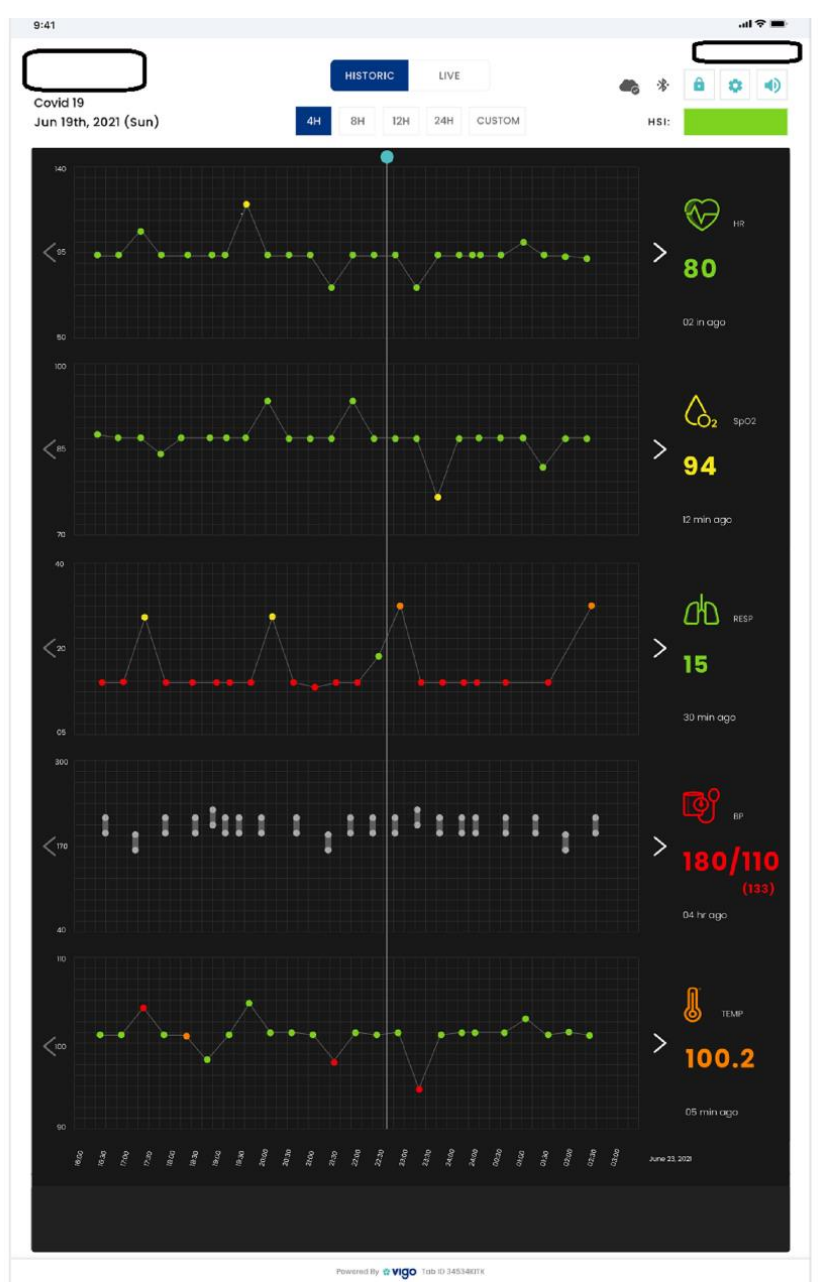

Fig.2 Multi Vital Monitoring Display

At ESIC Hyderabad, a retrospective research of RPM was undertaken with a sample size of 152 covid patients. The study's whole framework and methods were explained to the survey participants in their native languages, and informed consent was signed and taken. For the [ $n=152]$ covid19 positive cases with varying degrees of symptom severity, a remote patient monitoring tool was used. With the help of an RT-PCR report, the covid positive was confirmed. The patient's onboarding procedure is broken down into several basic steps. The patients were given vigo MVM solution and a clinical grade pulse 
oximeter. The skin temperature, respiration rate, and heart rate may all be measured with the vigo patch with biosensor. The vigo MVM solution is an FDA-approved gadget for detecting accurate vitals values. Each patch for the vigo MVM solution has its own patch code. The patient must enter the patch ID into the vigocare mobile application to connect the patch to the app. The vigocare application will use a hotspot to connect to the MVM solution patch. The patch begins gathering critical data and uploading it to the Vigo engine as soon as the connection is established. The Thevigo MVM solution will be integrated with the vigocare android app. The Vigo engine is a software interface that analyses vitals data and displays vital patterns in the form of color-coded graphs.

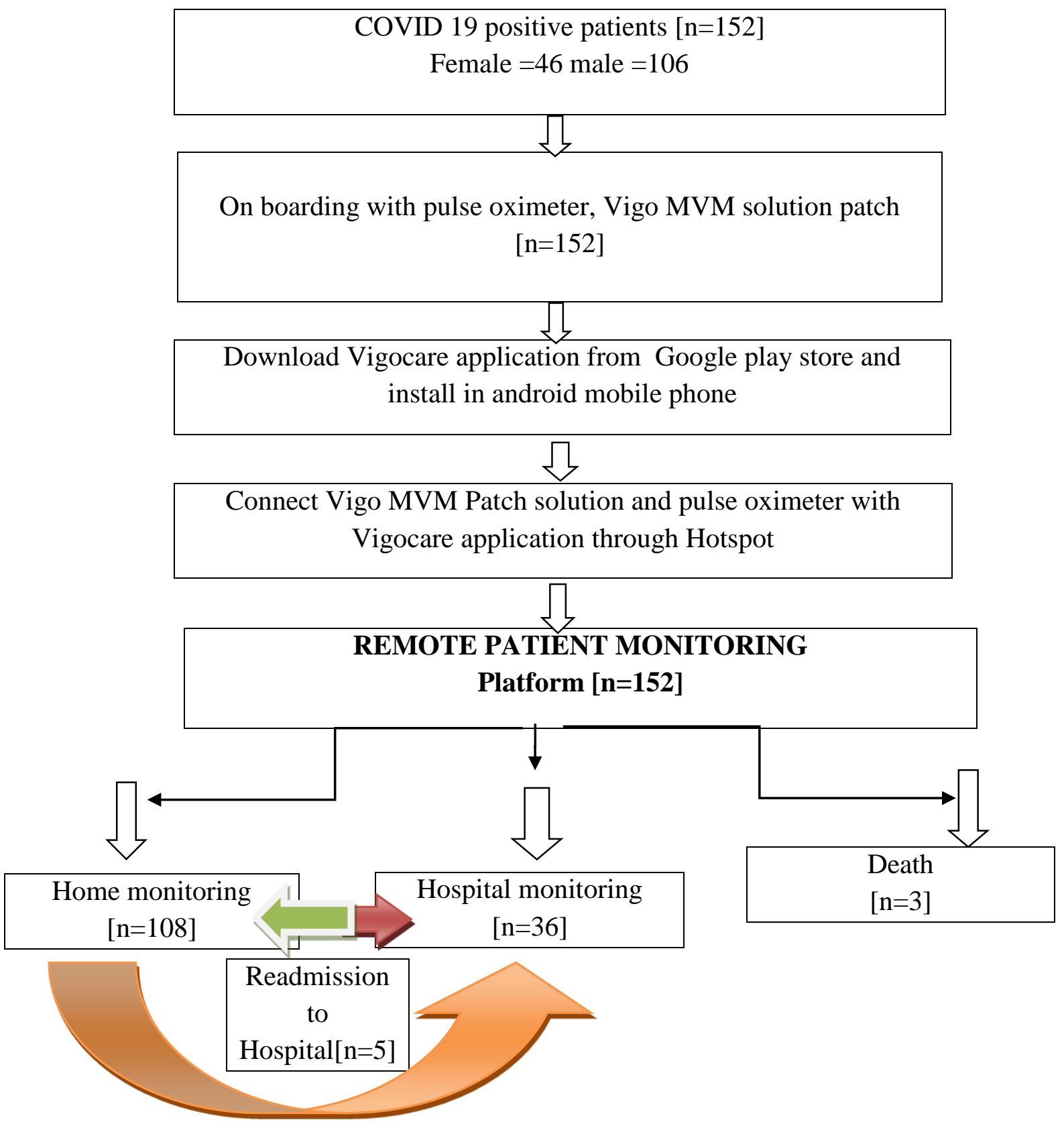

Fig.2 Schematic diagram of Remote Patient Monitoring [RPM] with Vigo MVM Solution. 
The green coloring in the graph indicates that the vitals are within the normal range and that the patient's condition is normal, while yellow suggests that some of the vitals may be deviating from normalcy, and red indicates that the majority of the vitals require immediate medical action. The central monitoring team would contact the allocated doctor or nurse right away to inform them of the vitals deviation. The physician will take prompt and appropriate action, saving the patient from further clinical deterioration

\section{Results and Discussions}

There were 46 women and 106 men among the 152 patients in the sample. The patients were first put on MVM solution and pulse oximeter for continuous remote patient monitoring for 5 days, and 98 patients were switched to home monitoring from the hospital owing to effective management and therapy. In the first five days, there were 33 females and 65 males. 90.7 percent of all home monitoring patients are in this category. Patients 7 and 3 were sent to home monitoring on the tenth and fifteenth days, respectively.

There were 108 home monitoring patients in total, accounting for 71.05 percent of the total research participants. A total of 36 patients were kept in hospital for additional treatment monitoring, with 5 females and 31 males accounting for $23 \%$ of the total.In all, 5 readmissions to the hospital occurred, with 3 females and 2 men accounting for 3.2 percent of the total. The death rate was 3 out of every 1,000 people, or 1.9 percent. It's possible that the fatality was caused by Covid19 or that the comorbid illnesses were exaggerated. Because the patients' medical history was not included in the research, the fatality rate might be questioned. Only 3.2 percent of patients required readmission to the emergency room, and the death rate was 1.8 percent. The medical histories of the patients were not taken into account in the study due to a lack of data. Exaggeration of comorbid diseases or cobid19 symptoms might be the cause of death.

According to the above research, $91 \%$ of hospital monitoring patients were transferred to home monitoring during the first five days, and readmissions were just 3.2 percent. Remote patient monitoring was shown to be a helpful choice for Covid19 patients. Patients in hospital surveillance were also placed on the Remote Monitoring MVM programme, which allows physicians and nursing personnel to avoid personal contact to the virus.

3.1 Trends analysis: Color indicators help physicians save time by allowing them to take the essential actions in patient medication, which is especially important for asymptomatic patients. The asymptomatic patient appears normal, but his or her saturation levels continue to decline, putting him or her in a potentially dangerous situation. vigocare RPM colour indicators can help handle this efficiently

\section{Conclusions}

The telehealth technology acknowledged by the European parliament and published in European parliament research services that includes remote patient monitoring technology. For low-income, densely populated South Asian nations like India, the RPM would be an 
alternate technique for managing pandemics. The RMP program was successful in treating COVID19 individuals and can be utilized to treat large groups of people.

The study had a modest number of participants. India is a diverse country with several ethnic groupings, as well as geographical and genetic pools. A full-fledged, welldocumented clinical trial may be required before remote patient monitoring may be used on a large scale.

\section{Acknowledgments}

We appreciate ESIC Medical College, Hospital staff and Vigocare management for the technological assistance and support in conducting the study. All the authors are contributed accordingly for writing and finalizing the manuscript for publication.

\section{Future scope}

The Innovative healthcare solutions like tele-medicine would save India between \$4-5 billion every year and replace half of the inpatient consultations as per the Union Minister of state for science and technology-India.

\section{Conflict of interest:}

The Authors declares that there is no conflict of interest.

\section{References}

[1]. Mantena $S$, Keshavjee S. Strengthening healthcare delivery with remote patient monitoring in the time of COVID-19. BMJ Heal Care Inf [Internet]. 2021;28:100302. Available from: http://informatics.bmj.com/

[2]. Kodama R, Arora S, Anand S, Choudhary A, Weingarten J, Francesco N, et al. Reengineering the Discharge Transition Process of COVID-19 Patients Using Telemedicine, Remote Patient Monitoring, and Around-the-Clock Remote Patient Monitoring from the Emergency Department and Inpatient Units. Available from: www.liebertpub.com

[3]. Lotfi M, Hamblin MR, Rezaei N. COVID-19: Transmission, prevention, and potential therapeutic opportunities. 2020 [cited 2021 Sep 13]; Available from: https://doi.org/10.1016/j.cca.2020.05.044

[4]. Vindrola-Padros C, Sidhu MS, Georghiou T, Sherlaw-Johnson C, Singh KE, Tomini SM, et al. The implementation of remote home monitoring models during the COVID-19 pandemic in England. EClinicalMedicine. 2021 Apr 1;34.

[5]. Who. Changes from the previous version. 2020; Available from: https://www.who.int/publications/i/item/considerations-in-adjusting-public-health-andsocial-measures-in-the-context-of-covid-19-interim-guidance

[6]. Mihalis K. Ten technologies to fight coronavirus. Eur Parliam Res Serv [Internet]. 2020;[April]:1-20. Available from: https://www.europarl.europa.eu/RegData/etudes/IDAN/2020/641543/EPRS_IDA[2020]6 
41543_EN.pdf

[7]. Tabacof L, Kellner C, Breyman E, Dewil S, Braren S, Nasr L, et al. Remote Patient Monitoring for Home Management of Coronavirus Disease 2019 in New York: A CrossSectional Observational Study. Telemed e-Health. 2021;27[6]:641-8.

[8]. Coffey JD, Christopherson LA, Glasgow AE, Pearson KK, Brown JK, Gathje SR, et al. ARTICLE Implementation of a multisite, interdisciplinary remote patient monitoring program for ambulatory management of patients with COVID-19. Available from: https://doi.org/10.1038/s41746-021-00490-9

[9]. Effect of telehealth on use of secondary care and mortality: findings from the Whole System Demonstrator cluster randomised trial. Available from: http://www.bmj.com/

[10]. Muller AE, Berg RC, Jardim PSJ, Johansen TB, Ormstad SS. Can Remote Patient Monitoring Be the New Standard in Primary Care of Chronic Diseases, Post-COVID-19? Telemed e-Health. 2021;00[00]:1-28.

[11]. Attia ZI, Kapa S, Noseworthy PA, Lopez-Jimenez F, Friedman PA. Artificial Intelligence ECG to Detect Left Ventricular Dysfunction in COVID-19: A Case Series. Mayo Clin Proc. 2020 Nov 1;95[11]:2464-6.

[12]. Gordon WJ, Henderson D, Desharone A, Fisher HN, Judge J, Levine DM, et al. Remote Patient Monitoring Program for Hospital Discharged COVID-19 Patients. Appl Clin Inform. 2020;11[5]:792-801.

[13]. Siam, Ali I et al Secure Health Monitoring Communication Systems Based on IoT and Cloud Computing for Medical Emergency Applications Hindawi Computational Intelligence and Neuroscience Volume 2021, Article ID 8016525, 23 pages https://doi.org/10.1155/2021/8016525 